\title{
Multi-Symplectic, Lagrangian, One-Dimensional Gas Dynamics
}

G. M. Webb $1, a)$

${ }^{1}$ CSPAR, The University of Alabama in Huntsville, Huntsville AL 35805, USA

(Dated: 11 September 2018)

The equations of Lagrangian, ideal, one-dimensional (1D), compressible gas dynamics are written in a multi-symplectic form using the Lagrangian mass coordinate $m$ and time $t$ as independent variables, and in which the Eulerian position of the fluid element $x=x(m, t)$ is one of the dependent variables. This approach differs from the Eulerian, multi-symplectic approach using Clebsch variables. Lagrangian constraints are used to specify equations for $x_{m}, x_{t}$ and $S_{t}$ consistent with the Lagrangian map, where $S$ is the entropy of the gas. We require $S_{t}=0$ corresponding to advection of the entropy $S$ with the flow. We show that the Lagrangian Hamiltonian equations are related to the de Donder-Weyl multi-momentum formulation. The pullback conservation laws and the symplecticity conservation laws are discussed. The pullback conservation laws correspond to invariance of the action with respect to translations in time (energy conservation) and translations in $m$ in Noether's theorem. The conservation law due to $m$-translation invariance gives rise to a novel nonlocal conservation law involving the Clebsch variable $r$ used to impose $\partial S(m, t) / \partial t=0$. Translation invariance with respect to $x$ in Noether's theorem is associated with momentum conservation. We obtain the Cartan-Poincaré form for the system, and use it to obtain a closed ideal of two-forms representing the equation system.

PACS numbers: 47.10.Df, 47.10.A, 47.10.ab, 45.20Jj, 0.230.Jr

a) gmw0002@uah.edu 


\section{INTRODUCTION}

In a recent paper, Webb et al. (2014d) obtained a multi-symplectic formulation of magnetohydrodynamics (MHD) by using Clebsch variables in an Eulerian variational principle, in which the Lagrangian is modified by constraints using Lagrange multipliers, that ensure mass, entropy, magnetic flux and the Lin constraint are conserved following the flow. The work of Webb et al. (2014c) used similar methods to Cotter et al. (2007) who derived multisymplectic equations for fluid dynamic type systems. The work of Cotter et al. (2007) uses the Euler-Poincaré approach to Hamiltonian fluid type systems developed by Holm et al. (1998). Webb and Mace (2015) derive a generalized potential vorticity type conservation law in MHD by using Noether's second theorem, in conjunction with a non-field aligned fluid relabelling symmetry.

Work on multi-momentum Hamiltonian systems has a long history, going back to the work of de Donder (1935) and Weul (1935) where multi-momentum maps and generalized Legendre transformations were introduced to generalize Hamiltonian mechanics to a more covariant formulation, where time in a fixed reference frame is not the only evolution variable in the equations. The de Donder-Weyl Hamiltonian equations apply to action principles in which the Lagrangian $L=L\left(\mathbf{x}, \varphi^{i}, \partial \varphi^{i} / \partial x^{\mu}\right)$ in the independent variables $\mathbf{x}$ and the dependent field variables $\varphi^{i}\left(1 \leq i \leq m\right.$ say), includes at least two independent derivatives $\partial \varphi^{k} / \partial x^{1}$ and $\partial \varphi^{k} / \partial x^{2}$ say, for $\varphi^{k}$. It turns out that the equations of ideal, 1D Lagrangian gas dynamics can be cast in the de Donder-Weyl Hamiltonian form, since the Lagrangian $L$ in the formulation depends on both $x_{m}$ and $x_{t}$ where $x=x(m, t)$ defines the Lagrangian map, in which $x$ is a function of the Lagrangian mass coordinate $m$ and time $t$. The de Donder-Weyl Hamiltonian then depends on the multi-momenta $\pi_{x}^{t}=\partial L / \partial x_{t}, \pi_{x}^{m}=\partial L / \partial x_{m}, \pi_{S}^{t}=\partial L / \partial S_{t}$ ( e.g. Kanatchikov (1997, 1998), Forger et al. (2013), Forger and Romero (2005)). There is an extensive literature on multi-symplectic and multi-momentum Hamiltonian systems (see e.g. Gotau (1991a.b), Gotay et al. (2004a.b), Roman-Rou (2009), Marsden and Shkoller (1999), Carinena et al. (1991), Bridges et al. (2005, 2010), Kanatchikou (1993, 1997, 1998), Cantrijn et al. (1999)).

Bridges et al. (2010) relate multi-symplectic structures and generalized Hamiltonian differential equation systems to the variational bicomplex. The system of equations is written in the generalized Hamiltonian form $\mathbf{X}\lrcorner \omega=-d H$ where $\mathbf{X}$ is a generalized Hamiltonian 
vector field, $\omega$ is the symplectic form, and $H$ is the generalized Hamiltonian. In this development $H$ can be a differential form (i.e. $H$ need not be a scalar) and $\mathbf{X}$ can be a multi-vector. This approach circumvents the need to use a Legendre transformation relating the Lagrangian to the Hamiltonian of the system, which is not always obvious for the case of singular Lagrangians. In the variational bicomplex, the exterior derivative $d$ is split up into horizontal $\left(d_{h}\right)$ and vertical $\left(d_{v}\right)$ components (i.e. $\left.d=d_{h}+d_{v}\right)$. The horizontal component of $d_{h} f$ for a function $f$ involving $\mathbf{u}$ and their derivatives and associated contact one-forms and tensors (i.e. the jet space), corresponds to changes on the solution manifold where the $\mathbf{u}$ explicitly depend on the independent variables $\mathbf{x}$. The vertical component $d_{v} f$ corresponds to changes in the contact one-forms in the jet space which are non-zero off the solution manifold (but are zero on the solution manifold). Both $d_{h}$ and $d_{v}$ are needed to compute higher order derivatives and differential forms and multi-vectors.

For the case of singular Lagrangians, Dirac's theory of constraints is useful in determining the Hamiltonian and a new Poisson bracket (the Dirac bracket) that satisfies the Jacobi identity (e.g. Chandre (2013), Chandre et al. (2013)).

One of the benifits of the multi-symplectic formulation of the fluid equations is that both space and time can be thought of as evolution variables (e.g. Bridges et al. $(\mathbf{2 0 0 5}))$ ). This is useful in the formulation of travelling wave problems, where two distinct Hamiltonian formulations of the equations are possible with, distinct Hamiltonians (e.g. Bridges (1992), Webb et al. (2005, 2007, 2008, 2014d)). For systems in one Cartesian space variable $x$ and one time variable $t$, both $x$ and $t$ can be regarded as the evolution variable. In the case that time is regarded as the evolution variable, the Hamiltonian corresponds to the conserved energy flux, in which the conserved $x$-momentum flux acts as a constraint. However, in the case that space variable $x$ is the evolution variable, the conserved momentum flux is the Hamiltonian and the conserved energy flux acts as a constraint. One of the original motivations for the multi-symplectic approach to field theory, was the desire for a covariant Hamiltonian formulation that is frame independent (e.g. Gotay et al. $(2004 \mathrm{a}, \mathrm{b}))$. In the usual Hamiltonian approach, the space and time variables are first chosen, and the equations are written in Hamiltonian form, in which time is the evolution variable. This leads to non-covariant evolution equations. Multi-symplectic Poisson bracket 
formulations of multi-symplectic systems have been developed for example, by (Kanatchiko (1993, 1997), Forger et al. (2013); Forger and Romero (2005)).

Our main aim is to obtain multi-symplectic and multi-momentum equations in fluid dynamics by working directly with the Lagrangian fluid dynamics equations (see e.g. Courant and Friederichs (1976), Sjöberg and Mahomed (2004) for 1D gas dynamics and Newcomb (1962) for the case of magnetohydrodynamics). Akhatov et al. (1991) and Bluman et al. (2010) give comprehensive accounts of planar gas dynamics, and the associated conservation laws of the equations using potential symmetries of the equations and related equations (the so-called tree of equations related to the gas dynamic equations) using both the Eulerian and Lagrangian form of the equations. Verosky (1984) studied higher order symmetries and recursion operators for symmetries of the 1D, isentropic gas dynamic equations. Nutku (1987) and Olver and Nutku (1988) have studied the bi-Hamiltonian and tri-Hamiltonian structure of the 1D gas dynamic equations for the case of an isentropic gas ( $S=$ const.).

The outline of the paper is as follows. In section 2, we present the equations of 1D Eulerian gas dynamics, including a discussion of the first law of thermodynamics. In Section 3, we introduce the Lagrangian map for 1D gas dynamics and write the equations in terms of the Lagrangian mass coordinate $m$ and time $t$ as independent variables where $x=x(m, t)$ gives the Eulerian position of the fluid element. A Lagrangian variational principle (e.g Broer and Kobussen (1974), Webb and Zank (2009)) without constraints is used to describe the system. The Euler Lagrange equations for the system give a nonlinear wave equation for $x=x(m, t)$ in which the entropy $S=S(m)$ is a pre-specified function of $m$. In Section 4 , we develop a constrained variational principle using $m$ and $t$ as independent variables, in which the Lagrangian fluid dynamic equations $x_{m}=\tau(\tau=1 / \rho), x_{t}=u$, and $S_{t}=0$ are treated as constraints. To ensure that $\partial S(m, t) / \partial t=0$ we add a constraint term $r \partial S / \partial t$ to the Lagrangian. Variations of the action with respect to $x$ gives the Lagrangian momentum equation. The variational principle requires that $\partial r(m, t) / \partial t=-T$ where $T$ is the temperature of the gas. This formulation is used to derive a de Donder-Weyl, multimomentum Hamiltonian description of the system (Section 4.1). In Section 4.2 we derive the multi-symplectic form of the equations, which uses the same dependent variables as the de Donder-Weyl formulation. Section 4.3 derives the pullback and symplecticity conservation laws (e.g. Hydon (2005)) for the system. It turns out that one of the pullback conservation laws (which corresponds to translation invariance of the action with respect to the Lagrange 
label $m$ ) yields a nonlocal conservation law involving the Clebsch variable $r$. In Section 4.4 we show how the nonlocal pullback conservation law, and the momentum and energy conservation laws arise from Noether's theorem. Section 4.5 presents the Cartan-Poincaré form for the system. The Cartan-Poincaré form can be used to obtain a closed ideal of forms representing the partial differential equation system. This set of forms can be used to obtain the Lie symmetries and conservation laws of the system using Cartan's geometric theory of partial differential equations (e.g. Harrison and Estabrook (1971); Wahlquist and Estabrook (1975)). It remains an open problem, to investigate in detail the closed ideal of forms obtained from the Cartan Poincaré form. Such an investigation will depend on the detailed form of the equation of state for the system (i.e. the dependence of the pressure $p$ and energy density $\varepsilon$ on the density $\rho$ and entropy $S$ of the system). This will be useful in extending the many investigations of 1D gas dynamics for the barotropic gas case, where $p=p(\rho)$, and $\varepsilon=\varepsilon(\rho)$, to the more general, non-barotopic case. Section 5 concludes with a summary and discussion.

\section{ONE DIMENSIONAL GAS DYNAMICS}

The time dependent, ideal, inviscid equations of Eulerian gas dynamics in one Cartesian space dimension can be written in the form (Courant and Friederichs (1976), Sjöberg and Mahomed (2004)):

$$
\begin{aligned}
\frac{\partial \rho}{\partial t}+u \frac{\partial \rho}{\partial x}+\rho \frac{\partial u}{\partial x} & =0, \\
\rho\left(\frac{\partial u}{\partial t}+u \frac{\partial u}{\partial x}\right)+\frac{\partial p}{\partial x} & =0, \\
\frac{\partial S}{\partial t}+u \frac{\partial S}{\partial x} & =0,
\end{aligned}
$$

where $u$ is the fluid velocity (assumed to be directed along the $x$-axis), $\rho$ is the gas density and $p$ is the gas pressure, and $S$ is the gas entropy. Equations (2.1)-(2.3) need to be supplemented by an equation of state for the gas, (e.g. $p=p(\rho, S))$. The equation of state of the gas is related to the first law of thermodynamics:

$$
T d S=d Q=d U+p d \tau \quad \text { where } \quad \tau=\frac{1}{\rho} .
$$


For ideal gas dynamics, $d Q / d t=0$ and $d S / d t=0$ where $d / d t=\partial / \partial t+u \partial / \partial x$ is the Lagrangian time derivative moving with the flow. In (2.4) $U$ is the internal energy of the gas per unit mass, $\tau=1 / \rho$ is the specific volume, and $T$ is the temperature of the gas. Using the internal energy per unit volume, $\varepsilon=\rho U$ instead of $U$, the first law of thermodynamics reduces to:

$$
T d S=\frac{1}{\rho}(d \varepsilon-w d \rho) \quad \text { where } \quad w=\frac{\varepsilon+p}{\rho},
$$

is the enthalpy of the gas. Assuming $\varepsilon=\varepsilon(\rho, S)$, (2.5) gives:

$$
\rho T=\varepsilon_{S}, \quad w=\varepsilon_{\rho}, \quad p=\rho \varepsilon_{\rho}-\varepsilon
$$

From (2.5):

$$
T d S=d w-\frac{1}{\rho} d p \quad \text { and } \quad-\frac{1}{\rho} \nabla p=T \nabla S-\nabla w,
$$

which is useful in obtaining the Eulerian energy conservation equation for the system.

\section{THE LAGRANGIAN MAP}

In fluid dynamics, the Lagrangian map is defined as the solution of the differential equation system (e.g Courant and Friederichs (1976), Broer and Kobussen (1974)):

$$
\frac{d \mathbf{x}}{d t}=\mathbf{u}(\mathbf{x}, t)
$$

of the form $\mathbf{x}=\mathbf{X}\left(\mathbf{x}_{0}, t\right)$ where $\mathbf{x}=\mathbf{x}_{0}$ at time $t=0$, in which the fluid velocity $\mathbf{u}(\mathbf{x}, t)$ is assumed to be a known function of $\mathbf{x}$ and $t$. If $\mathbf{x}=\mathbf{X}\left(\mathbf{x}_{0}, t\right)$ is a 1-1 invertible map, then the inverse map gives $\mathbf{x}_{0}=\mathbf{f}(\mathbf{x}, t)$ and the fluid velocity $\mathbf{u}$ is given by $\mathbf{u}=\partial \mathbf{X}\left(\mathbf{x}_{0}, t\right) / \partial t$, where the time derivative is taken with the Lagrange label $\mathbf{x}_{0}$ held constant.

For the case of $1 \mathrm{D}$ gas dynamics in one Cartesian space coordinate $x$, the Lagrangian map implies:

$$
d x=\frac{\partial x}{\partial x_{0}} d x_{0}+\frac{\partial x}{\partial t} d t=\frac{\partial x}{\partial x_{0}}\left(\frac{\partial x_{0}}{\partial x} d x+\frac{\partial x_{0}}{\partial t} d t\right)+\frac{\partial x}{\partial t} d t
$$

which implies:

$$
\frac{\partial x}{\partial x_{0}} \frac{\partial x_{0}}{\partial x}=1, \quad \frac{\partial x}{\partial t}+\frac{\partial x}{\partial x_{0}} \frac{\partial x_{0}}{\partial t}=0
$$


From (3.3) we obtain:

$$
\frac{\partial x_{0}}{\partial t}+u \frac{\partial x_{0}}{\partial x}=0
$$

and hence the Lagrange label $x_{0}$ is advected with the flow.

The mass continuity equation (2.1) may be written in the form:

$$
\rho(x, t) d x=\rho_{0} d x_{0} \quad \text { or } \quad \rho J=\rho_{0},
$$

where $\rho_{0}=\rho\left(x_{0}, 0\right)$ and $J=\partial x / \partial x_{0}$ is the Jacobian of the Lagrangian map. The Lagrangian mass coordinate:

$$
m=\int_{-\infty}^{x} \rho\left(x^{\prime}, t\right) d x^{\prime}=\int_{-\infty}^{x_{0}} \rho\left(x_{0}^{\prime}, 0\right) d x_{0}^{\prime},
$$

can be used instead of $x_{0}$ as a Lagrangian label. Note that $m=m\left(x_{0}\right)$ and the entropy $S=S\left(x_{0}\right)$ are both advected with the flow. Using the definition of the Lagrangian map and using (3.6) we obtain the auxiliary relations:

$$
x_{m}=\tau=\frac{1}{\rho} \text { and } \quad x_{t}=u
$$

where we regard $x=x(m, t)$ to be a function of $m$ and $t$.

A variational principle for 1D gas dynamics is well known (e.g. Courant and Friederichs (1976), Broer and Kobussen (1974), Webb and Zank (2009)). The action is defined as:

$$
A=\iint \mathcal{L} d x d t=\iint \mathcal{L}_{0} d m d t
$$

where $\mathcal{L}$ and $\mathcal{L}_{0}$ are defined by:

$$
\mathcal{L}=\frac{1}{2} \rho x_{t}^{2}-\varepsilon(\rho, S), \quad \mathcal{L}_{0}(\mathbf{x}, \mathbf{m}, \mathbf{t})=x_{m} \mathcal{L}=\frac{1}{2} x_{t}^{2}-F\left(x_{m}, m\right), \quad F\left(x_{m}, m\right)=\frac{\varepsilon(\rho, S)}{\rho}
$$

The Lagrangian $x$-momentum equation for the system is given by the variational equation $\delta A / \delta x=0$, i.e.

$$
\frac{\delta A}{\delta x}=-\left(x_{t t}+p_{m}\right)=0 \quad \text { where } \quad p=\rho \varepsilon_{\rho}-\varepsilon=-\frac{\partial F\left(x_{m}, m\right)}{\partial x_{m}}
$$

is the gas pressure. The Eulerian momentum equation $u_{t}+u u_{x}=-p_{x} / \rho$ is obtained by 
multiplying the Lagrangian momentum equation (3.10) by $\rho=1 / x_{m}$ (note $x_{t t}=u_{t}+u u_{x}$ ).

The Euler Lagrange equation (3.10) is in fact a nonlinear wave equation for $x(m, t)$ of the form:

$$
x_{t t}-a^{2} \frac{x_{m m}}{x_{m}^{2}}+p_{S} S_{m}=0,
$$

where $a^{2}=(\partial p / \partial \rho)_{S}$ is the square of the adiabatic sound speed of the gas. For the special case of a gas with a constant adiabatic index $\gamma$, the gas pressure has the form:

$$
p=p_{1}\left(\frac{\rho}{\rho_{1}}\right)^{\gamma} \exp \left(\frac{S}{C_{v}}\right) \quad \text { and } \quad \rho=\frac{1}{x_{m}}
$$

In this latter case (3.11) reduces to the equation:

$$
x_{t t}=N_{1} x_{m}^{-\gamma-1} \exp [\bar{S}(m)]\left(\gamma x_{m m}-x_{m} \bar{S}_{m}\right)
$$

where $N_{1}=p_{1} \rho_{1}^{-\gamma}$ and $\bar{S}=S / C_{v}$. This is a complicated, nonlinear wave equation for $x(m, t)$. However, it reduces to a linear elliptic equation for the case of the Chaplygin gas with $\gamma=-1$ (e.g. Akhatov et al. (1991), Webb and Zank (2009)).

Using the first law of thermodynamics (2.5)-(2.7) we obtain the Lagrangian evolution equation for the gas pressure as:

$$
p_{t}+\frac{a^{2}}{\tau^{2}} u_{m}=0
$$

where $p=p(m, t)$ is the Lagrangian form for $p$. In the Lagrangian fluid description, the mass continuity equation takes the form:

$$
u_{m}=\tau_{t}
$$

The mass continuity equation (3.15) is a consequence of the integrability condition $x_{m t}=x_{t m}$ in the Lagrangian approach.

\section{DE DONDER-WEYL AND MULTI-SYMPLECTIC APPROACH}

Consider the action:

$$
\hat{A}=\int \hat{L} d t d m
$$


where the Lagrangian density $\hat{\mathbf{L}}(\mathbf{x}, \mathbf{t}, \mathbf{m}, \mathbf{u}, \tau, \mathbf{S}, \mathbf{r})$ is given by:

$$
\hat{L}=L+\lambda\left(x_{m}-\tau\right)+\nu\left(x_{t}-u\right)+r S_{t}, \quad L=\frac{1}{2} u^{2}-\varepsilon \tau .
$$

The constrained variational principle (4.1)-(4.2) with Lagrange multipliers $\lambda, \nu$ and $r$ ensures that the basic $1 \mathrm{D}$ gas dynamic equations:

$$
x_{m}-\tau=0, \quad x_{t}-u=0 \quad \text { and } \quad S_{t}=0,
$$

are satisfied. Here, $L$ is the unconstrained Lagrangian density for $1 \mathrm{D}$ gas dynamics, i.e. $L=\mathcal{L}_{0}$ where $\mathcal{L}_{0}$ is given by $(\underline{3.9})$.

For the variational principle (4.1)-(4.2) the stationary point conditions for the action give the equations:

$$
\begin{aligned}
& \frac{\delta \hat{A}}{\delta \tau}=\rho \varepsilon_{\rho}-\varepsilon-\lambda \equiv(p-\lambda)=0, \quad \frac{\delta \hat{A}}{\delta \lambda}=\left(x_{m}-\tau\right)=0 \\
& \frac{\delta \hat{A}}{\delta u}=(u-\nu)=0, \quad \frac{\delta \hat{A}}{\delta \nu}=\left(x_{t}-u\right)=0 \\
& \frac{\delta \hat{A}}{\delta S}=-\left(r_{t}+T\right)=0, \quad \frac{\delta \hat{A}}{\delta r}=S_{t}=0 .
\end{aligned}
$$

The variational equation:

$$
\frac{\delta \hat{A}}{\delta x}=-\left(\nu_{t}+\lambda_{m}\right)=-\left(u_{t}+p_{m}\right)=0
$$

gives the Lagrangian momentum equation for the system.

Equations (4.4) and (4.5) give

$$
\lambda=p \quad \text { and } \quad \nu=u
$$

for the Lagrange multipliers in (4.2). Thus, we can replace $\hat{L}$ in (4.1) by:

$$
\begin{aligned}
\tilde{L}(x, t, m, u, p, r, S) & =\frac{1}{2} u^{2}-\frac{\varepsilon}{\rho}+p\left(x_{m}-\tau\right)+u\left(x_{t}-u\right)+r S_{t} \\
& =u x_{t}+p x_{m}+r S_{t}-\left(\frac{1}{2} u^{2}+w\right)
\end{aligned}
$$


where $w=(\varepsilon+p) / \rho$ is the enthalpy of the gas and $\tau=1 / \rho$. In (4.9) the Lagrange multiplier $r$ ensures that the entropy is advected with the flow, i.e. $S_{t}=0$ where $S=S(m, t)$. In our analysis we use the state vector:

$$
\mathbf{z}=(x, u, p, S, r)^{T}
$$

The internal energy density $\varepsilon=\varepsilon(\rho, S), p=p(\rho, S)$ and $w=w(\rho, S)$. However, we prefer to use $p$ and $S$ as independent variables to specify the state of the gas. We introduce the function $\tilde{w}(p, S)=w(\rho, S)$ in our analysis. By equating $d \tilde{w}(p, S)$ and $d w(\rho, S)$ we obtain the partial differential relations:

$$
\tilde{w}_{p} a^{2}=w_{\rho}, \quad \tilde{w}_{p} p_{S}+\tilde{w}_{S}=w_{S}
$$

Using the thermodynamic relations (2.4)-(2.7) we obtain:

$$
\begin{aligned}
& \tilde{w}_{p}=\frac{w_{\rho}}{a^{2}}=\tau, \\
& \tilde{w}_{S}=w_{S}-p_{S} \frac{w_{\rho}}{a^{2}}=\frac{\varepsilon_{S}}{\rho}=T
\end{aligned}
$$

where $T$ is the temperature of the gas.

Consider the variations of the action $\tilde{A}(4.1)$, but with $\hat{L}$ replaced by $\tilde{L}$. Using (4.9) for $\tilde{L}$ we obtain:

$$
\begin{aligned}
& \frac{\delta \tilde{A}}{\delta x}=-\left(u_{t}+p_{m}\right)=0, \quad \frac{\delta \tilde{A}}{\delta u}=\left(x_{t}-u\right)=0, \\
& \frac{\delta \tilde{A}}{\delta p}=x_{m}-\tilde{w}_{p}=\left(x_{m}-\tau\right)=0, \\
& \frac{\delta \tilde{A}}{\delta S}=-\tilde{w}_{S}-r_{t}=-\left(r_{t}+T\right)=0, \quad \frac{\delta \tilde{A}}{\delta r}=S_{t}=0 .
\end{aligned}
$$

Thus, the variational equations (4.4)-(4.8) give the same variational equations as (4.13) based on the variational derivatives of $\tilde{A}$ and $\tilde{L}$. Both approaches give the basic equations of 1D Lagrangian gas dynamics, namely:

$$
u_{t}+p_{m}=0, \quad x_{t}=u, \quad x_{m}=\tau, \quad S_{t}=0, \quad r_{t}+T=0 .
$$


The equation for the Lagrange multiplier $r$ is similar to the Lagrange multiplier equation for $\beta / \rho$ in the Eulerian Clebsch variable formulation (e.g. Zakharov and Kuznetsov (1997), Morrison (1998), Holm and Kupershmidt (1983a,b), Webb et al. (2014c)). Note that $S_{t}=0$ following the flow implies $S=S(m)$. A similar variational principle to (4.9) was used by Webb and Zank (2009) in a paper on conservation laws in 1D gas dynamics (equation (6.20) of that paper).

\section{A. The de Donder-Weyl formulation}

A general description of the de-Donder Weyl equations and multi-symplectic geometry has been given for example by Paufler and Römer (2002), who use the language of fiber bundles. We present an elementary derivation of the general form of the de Donder-Weyl Hamiltonian equations below. The basic results concern an action principle of the form:

$$
J=\int \mathcal{L}\left(x^{\mu}, \varphi^{i}, \partial_{\mu} \varphi^{i}\right) d \mathbf{x}
$$

where the $x^{\mu}$ are the independent variables, and the $\varphi^{i}$ are the dependent field variables. The Euler Lagrange equations for the variational principle (4.15) are:

$$
\frac{\delta J}{\delta \varphi^{i}}=\frac{\partial \mathcal{L}}{\partial \varphi^{i}}-\frac{\partial}{\partial x^{\mu}}\left(\frac{\partial \mathcal{L}}{\partial\left(\partial_{\mu} \varphi^{i}\right)}\right)=0 .
$$

The poly-momenta for the system are defined as:

$$
\pi_{i}^{\mu}=\frac{\partial \mathcal{L}}{\partial\left(\partial_{\mu} \varphi^{i}\right)}
$$

In the Hamiltonian description, the multi-symplectic Hamiltonian $H\left(x^{\mu}, \varphi^{i}, \pi_{i}^{\mu}\right)$ is governed by the generalized Legendre transformation:

$$
H=\pi_{i}^{\mu} \frac{\partial \varphi^{i}}{\partial x^{\mu}}-\mathcal{L}
$$


Proceeding as in classical mechanics (e.g. Goldstein (1980)), one obtains the balance equations:

$$
\begin{aligned}
d H & =d \pi_{i}^{\mu} \varphi_{, \mu}^{i}+\pi_{i}^{\mu} d\left(\varphi_{, \mu}^{i}\right)-\left(\frac{\partial \mathcal{L}}{\partial x^{\mu}} d x^{\mu}+\frac{\partial \mathcal{L}}{\partial \varphi^{i}} d \varphi^{i}+\frac{\partial \mathcal{L}}{\partial\left(\varphi_{, \mu}^{i}\right)} d \varphi_{, \mu}^{i}\right) \\
& =\frac{\partial H}{\partial x^{\mu}} d x^{\mu}+\frac{\partial H}{\partial \varphi^{i}} d \varphi^{i}+\frac{\partial H}{\partial \pi_{i}^{\mu}} d \pi_{i}^{\mu} .
\end{aligned}
$$

Equating the coefficients of $d x^{\mu}, d \pi_{i}^{\mu}, d \varphi_{, \mu}^{i}$ and $d \varphi^{i}$ gives the balance equations:

$$
\begin{aligned}
\frac{\partial H}{\partial x^{\mu}}+\frac{\partial \mathcal{L}}{\partial x^{\mu}} & =0 \\
\varphi_{, \mu}^{i}-\frac{\partial H}{\partial \pi_{i}^{\mu}} & =0 \\
\pi_{i}^{\mu}-\frac{\partial \mathcal{L}}{\partial \varphi_{, \mu}^{i}} & =0 \\
\frac{\partial \mathcal{L}}{\partial \varphi^{i}}+\frac{\partial H}{\partial \varphi^{i}} & =0 .
\end{aligned}
$$

Using the Euler Lagrange equations (4.16), the definitions (4.17) of the polymomenta $\pi_{i}^{\mu}$ and (4.21) and (4.23) we obtain the de Donder-Weyl Hamiltonian equations:

$$
\frac{\partial \varphi^{i}}{\partial x^{\mu}}=\frac{\partial H}{\partial \pi_{i}^{\mu}}, \quad \frac{\partial}{\partial x^{\mu}}\left(\pi_{i}^{\mu}\right)=-\frac{\partial H}{\partial \varphi^{i}},
$$

which are analogous to Hamilton's equations in ordinary Hamiltonian mechanics, in which the time $t$ is the evolution variable. A more complete description of the de Donder-Weyl equations using fiber bundles and jet bundles is given by Paufler and Römer (2002).

Below, we use the above approach to multi-symplectic systems, to determine the de Donder-Weyl equations for 1D Lagrangian gas dynamics. We introduce two momenta corresponding to $x(m, t)$ and a momentum associated with $S_{t}$ via the momentum maps:

$$
\pi_{x}^{t}=\frac{\partial \tilde{L}}{\partial x_{t}}, \quad \pi_{x}^{m}=\frac{\partial \tilde{L}}{\partial x_{m}} \quad \text { and } \quad \pi_{S}^{t}=\frac{\partial \tilde{L}}{\partial S_{t}}
$$

where the Lagrangian $\tilde{L}$ is given by (4.9). We find:

$$
\pi_{x}^{t}=\frac{\partial \tilde{L}}{\partial x_{t}}=u, \quad \pi_{x}^{m}=\frac{\partial \tilde{L}}{\partial x_{m}}=p, \quad \pi_{S}^{t}=\frac{\partial \tilde{L}}{\partial S_{t}}=r
$$


Introduce the Hamiltonian $H$ by the generalized Legendre transformation:

$$
\begin{aligned}
H & =\pi_{x}^{t} x_{t}+\pi_{x}^{m} x_{m}+\pi_{S}^{t} S_{t}-\tilde{L}, \\
& =u x_{t}+p x_{m}+r S_{t}-\left(-\frac{1}{2} u^{2}+u x_{t}+p x_{m}+r S_{t}-w\right) \\
& =\frac{1}{2} u^{2}+w
\end{aligned}
$$

where $w=(\varepsilon+p) / \rho$ is the gas enthalpy. The Hamiltonian (4.27) is the Eulerian energy flux per unit mass flux, i.e.

$$
\frac{\partial}{\partial t}\left[\frac{1}{2} \rho u^{2}+\varepsilon(\rho, S)\right]+\frac{\partial}{\partial x}[\rho u H]=0,
$$

is the Eulerian energy conservation equation. $H$ in (4.27) can be expressed solely in terms of the canonical momenta $\pi_{x}^{t}=u, \pi_{x}^{m}=p$ and $S$.

In the general de Donder-Weyl theory, the basic idea is the generalized Legendre transformation:

$$
H=\pi_{x}^{t} x_{t}+\pi_{x}^{m} x_{m}+\pi_{S}^{t} S_{t}-\tilde{L}\left(x, x_{t}, x_{m}, S, p, r, u, S_{t}\right),
$$

where the Hamiltonian density $H$ has the form:

$$
H=H\left(x, S, \pi_{x}^{t}, \pi_{x}^{m}, \pi_{S}^{t}\right) .
$$

From (4.29) and (4.30) we obtain:

$$
\begin{aligned}
d H= & \frac{\partial H}{\partial x} d x+\frac{\partial H}{\partial S} d S+\frac{\partial H}{\partial \pi_{x}^{t}} d \pi_{x}^{t}+\frac{\partial H}{\partial \pi_{x}^{m}} d \pi_{x}^{m}+\frac{\partial H}{\partial \pi_{S}^{t}} d \pi_{S}^{t} \\
= & \left(x_{t} d \pi_{x}^{t}+\pi_{x}^{t} d x_{t}+x_{m} d \pi_{x}^{m}+\pi_{x}^{m} d x_{m}+S_{t} d \pi_{S}^{t}+\pi_{S}^{t} d S_{t}\right) \\
& -\left(\frac{\partial \tilde{L}}{\partial x} d x+\frac{\partial \tilde{L}}{\partial x_{t}} d x_{t}+\frac{\partial \tilde{L}}{\partial x_{m}} d x_{m}+\frac{\partial \tilde{L}}{\partial S} d S+\frac{\partial \tilde{L}}{\partial S_{t}} d S_{t}+\frac{\partial \tilde{L}}{\partial u} d u+\frac{\partial \tilde{L}}{\partial r} d r+\frac{\partial \tilde{L}}{\partial p} d p\right) .
\end{aligned}
$$


From the Euler Lagrange equations (4.13) we have:

$$
\begin{aligned}
& \frac{\partial \tilde{L}}{\partial u}=\frac{\delta \tilde{A}}{\delta u}=x_{t}-u=0, \\
& \frac{\partial \tilde{L}}{\partial r}=\frac{\delta \tilde{A}}{\delta r}=S_{t}=0, \\
& \frac{\partial \tilde{L}}{\partial p}=\frac{\delta \tilde{A}}{\delta p}=x_{m}-\tau=0 .
\end{aligned}
$$

Equating coefficients of $d x_{t}, d x_{m}$, and $d S_{t}$ in (4.31) gives:

$$
\pi_{x}^{t}=\frac{\partial \tilde{L}}{\partial x_{t}}, \quad \pi_{x}^{m}=\frac{\partial \tilde{L}}{\partial x_{m}}, \quad \pi_{S}^{t}=\frac{\partial \tilde{L}}{\partial S_{t}}
$$

which are the usual definitions for the canonical momenta. Equating the coefficients of $d \pi_{x}^{t}$, $d \pi_{x}^{m}$ and $d \pi_{S}^{t}$ in (4.31) gives:

$$
x_{t}=\frac{\partial H}{\partial \pi_{x}^{t}}, \quad x_{m}=\frac{\partial H}{\partial \pi_{x}^{m}}, \quad S_{t}=\frac{\partial H}{\partial \pi_{S}^{t}},
$$

which are the canonical evolution equations of $x$ with respect to $t$ and $h$ and the evolution equation for $S$ with respect to time. Equating the coefficients of $d x$ and $d S$ equal to zero gives the equations:

$$
\frac{\partial \tilde{L}}{\partial x}+\frac{\partial H}{\partial x}=0 \text { and } \quad \frac{\partial \tilde{L}}{\partial S}+\frac{\partial H}{\partial S}=0
$$

In the present example $\partial \tilde{L} / \partial x=\partial H / \partial x=0$ and $\partial H / \partial S=\partial \tilde{w} / \partial S=T$ and $\partial \tilde{L} / \partial S=-T$, which verifies the validity of (4.35). The Euler Lagrange equation $\delta \tilde{A} / \delta x=0$ in (4.13) can be written in the form:

$$
\frac{\partial}{\partial t}\left(\frac{\partial \tilde{L}}{\partial x_{t}}\right)+\frac{\partial}{\partial m}\left(\frac{\partial \tilde{L}}{\partial x_{m}}\right)-\frac{\partial \tilde{L}}{\partial x}=0 \quad \text { or } \quad \frac{\partial \pi_{x}^{t}}{\partial t}+\frac{\partial \pi_{x}^{m}}{\partial h}=-\frac{\partial H}{\partial x}=0
$$

This equation is 'canonically conjugate' to the equations:

$$
\begin{gathered}
x_{t}=\frac{\partial H}{\partial \pi_{x}^{t}}=\frac{\partial H}{\partial u}=u, \\
x_{m}=\frac{\partial H}{\partial \pi_{x}^{m}}=\frac{\partial H}{\partial p}=\tau .
\end{gathered}
$$


The canonically conjugate equations for $\pi_{S}^{t}=r$ and $S$ are:

$$
\begin{aligned}
\frac{\partial \pi_{S}^{t}}{\partial t} & =-\frac{\partial H}{\partial S} \quad \text { or } \quad \frac{\partial r}{\partial t}=-T \\
\frac{\partial S}{\partial t} & =\frac{\partial H}{\partial r}=0 .
\end{aligned}
$$

Thus, the de Donder-Weyl formulation (4.36)-(4.38) are the generalization of Hamilton's equations for the multi-momentum case. These equations allow for canonical momenta associated with both the space and time (i.e. $m$ and $t$ ) gradients. Also (4.36) for the momentum $\boldsymbol{\pi}_{x}=\left(\pi_{x}^{t}, \pi_{x}^{m}\right)^{T}$ can be written in the form:

$$
\nabla \cdot \boldsymbol{\pi}_{x}=-\frac{\partial H}{\partial x} \quad \text { where } \quad \nabla=\left(\partial_{t}, \partial_{m}\right)^{T}
$$

is the gradient operator in $(t, m)$ space.

\section{B. Multi-symplectic approach}

Following Hydon (2005), we introduce the notation:

$$
\mathbf{z}=(x, u, p, S, r)^{T} \equiv\left(x, \pi_{x}^{t}, \pi_{x}^{m}, S, \pi_{S}^{t}\right)^{T}
$$

as the dependent variables describing the system. We introduce the one-forms:

$$
\omega^{\alpha}=L_{s}^{\alpha} d z^{s}, \quad \alpha=0,1, \quad s=1,2,
$$

where

$$
L_{s}^{\alpha} \frac{\partial z^{s}}{\partial x^{\alpha}}=u \frac{\partial x}{\partial t}+p \frac{\partial x}{\partial m}+r \frac{\partial S}{\partial t}
$$

are the terms in the Legendre transform (4.27) relating $H$ and $L$ (note the $L_{s}^{\alpha}$ are the canonical momenta in the de Donder-Weyl approach). In (4.42) $x^{0}=t$ and $x^{1}=m$. From (4.41)-(4.42) we identify the one forms:

$$
\omega^{0}=u d x+r d S \equiv L_{1}^{0} d x+L_{4}^{0} d S, \quad \omega^{1}=p d x \equiv L_{1}^{1} d x
$$

as the fundamental one forms describing the system. 
In multi-symplectic systems, the fundamental 2 -forms $\kappa^{\alpha}=d \omega^{\alpha}$ are given by:

$$
\kappa^{\alpha}=d \omega^{\alpha}=d\left(L_{j}^{\alpha} d z^{j}\right)=\frac{1}{2} K_{i j}^{\alpha} d z^{i} \wedge d z^{j}
$$

Thus,

$$
\mathbf{K}_{i j}^{\alpha}=\frac{\partial L_{j}^{\alpha}}{\partial z^{i}}-\frac{\partial L_{i}^{\alpha}}{\partial z^{j}}
$$

where the matrices $\mathrm{K}_{i j}^{\alpha}$ are skew symmetric with respect to the 2 lower indices (e.g. Hydon (2005), Cotter et al. (2007), Webb et al. (2014c)). The condition $\nabla_{\alpha} \kappa^{\alpha}=0$, corresponds to the symplecticity or conservation of phase space condition for multi-symplectic systems. Here $\nabla_{\alpha}$ denotes covariant differentiation with respect to the independent variables $x^{\alpha}$ (i.e. $m$ and $t)$.

Taking the exterior derivatives of $\omega^{0}$ and $\omega^{1}$ in (4.43) gives the 2-forms:

$$
\begin{aligned}
& d \omega^{0}=d u \wedge d x+d r \wedge d S=d z^{2} \wedge d z^{1}+d z^{5} \wedge d z^{4}=\frac{1}{2} \mathrm{~K}_{i j}^{0} d z^{i} \wedge d z^{j} \\
& d \omega^{1}=d p \wedge d x=d z^{3} \wedge d z^{1}=\frac{1}{2} \mathrm{~K}_{i j}^{1} d z^{i} \wedge d z^{j}
\end{aligned}
$$

Thus, we identify the non-zero components of the skew-symmetric matrices $\mathrm{K}_{i j}^{\alpha}$ as:

$$
\mathrm{K}_{21}^{0}=1, \quad \mathrm{~K}_{12}^{0}=-1, \quad \mathrm{~K}_{54}^{0}=1, \quad \mathrm{~K}_{45}^{0}=-1, \quad \mathrm{~K}_{31}^{1}=1, \quad \mathrm{~K}_{13}^{1}=-1 .
$$

From the general theory of multi-symplectic systems (Hydon (2005)) it follows that (4.14) can be written in the multi-symplectic form:

$$
\mathrm{K}_{i j}^{0} \frac{\partial z^{j}}{\partial t}+\mathrm{K}_{i j}^{1} \frac{\partial z^{j}}{\partial x}=\frac{\delta \mathcal{H}}{\delta z^{i}}
$$

where $\mathcal{H}$ is the Hamiltonian functional:

$$
\mathcal{H}=\int H d m
$$

In the present analysis

$$
\frac{\delta \mathcal{H}}{\delta \mathbf{z}}=\frac{\partial H}{\partial \mathbf{z}}=\left(\frac{\partial H}{\partial x}, \frac{\partial H}{\partial u}, \frac{\partial H}{\partial p}, \frac{\partial H}{\partial S}, \frac{\partial H}{\partial r}\right)^{T}=(0, u, \tau, T, 0)^{T}
$$


The multi-symplectic system (4.48) reduces to the matrix system

$$
\left(\begin{array}{ccccc}
0 & -1 & 0 & 0 & 0 \\
1 & 0 & 0 & 0 & 0 \\
0 & 0 & 0 & 0 & 0 \\
0 & 0 & 0 & 0 & -1 \\
0 & 0 & 0 & 1 & 0
\end{array}\right) \frac{\partial}{\partial t}\left(\begin{array}{c}
x \\
u \\
p \\
S \\
r
\end{array}\right)+\left(\begin{array}{ccccc}
0 & 0 & -1 & 0 & 0 \\
0 & 0 & 0 & 0 & 0 \\
1 & 0 & 0 & 0 & 0 \\
0 & 0 & 0 & 0 & 0 \\
0 & 0 & 0 & 0 & 0
\end{array}\right) \frac{\partial}{\partial m}\left(\begin{array}{c}
x \\
u \\
p \\
S \\
r
\end{array}\right)=\frac{\partial H}{\partial \mathbf{z}} .
$$

The component equations in (4.51) are:

$$
-\left(u_{t}+p_{m}\right)=0, \quad x_{t}=u, \quad x_{m}=\tau, \quad r_{t}=-T, \quad S_{t}=0
$$

which is the system (4.14) for $1 \mathrm{D}$, Lagrangian gas dynamics. Note that $\mathrm{K}_{i j}^{0}$ and $\mathrm{K}_{i j}^{1}$ are skew symmetric matrices.

\section{Pullback and symplecticity conservation laws}

From Hydon (2005) (see also Webb et al. (2014c) and Appendix A), the multi-symplectic system (4.51) satisfies the pullback conservation laws:

$$
D_{\alpha}\left(L_{j}^{\alpha} z_{, \beta}^{j}-L \delta_{\beta}^{\alpha}\right)=0, \quad \alpha, \beta=0,1
$$

where $\left(x^{0}, x^{1}\right)=(t, m)$ in the present application. The pullback conservation laws can also be derived from Noether's theorem and correspond to invariance of the action under translations of the independent variables (i.e. the $x^{\beta}$ ). In (4.53) the Lagrangian density $L$ is given by:

$$
L=\frac{1}{2} u^{2}-\varepsilon \tau
$$

where the Lagrangian $L$ is the same as in (4.2) except the constraint terms are omitted in (4.54). The pullback conservation laws are a consequence of the generalized Legendre transformation (e.g. Hydon (2005)).

For $\beta=0$, the pullback conservation law (4.53) reduces to:

$$
D_{t} I^{0}+D_{m} I^{1}=0
$$


where

$$
I^{0}=L_{j}^{0} z_{, 0}^{j}-L, \quad I^{1}=L_{j}^{1} z_{, 0}^{j} .
$$

Using (4.43) for $\omega^{0}$ and $\omega^{1}$ we obtain:

$$
\begin{aligned}
& I^{0}=u \frac{\partial x}{\partial t}+r \frac{\partial S}{\partial t}-\left(\frac{1}{2} u^{2}-\varepsilon \tau\right) \equiv \frac{1}{2} u^{2}+\varepsilon \tau \\
& I^{1}=p \frac{\partial x}{\partial t} \equiv p u
\end{aligned}
$$

Thus, we obtain the conservation law:

$$
\frac{\partial}{\partial t}\left(\frac{1}{2} u^{2}+\varepsilon \tau\right)+\frac{\partial}{\partial m}(p u)=0
$$

which is the co-moving energy equation. Using (3.6), we obtain:

$$
\frac{\partial}{\partial m}=\frac{\partial x_{0}}{\partial m} \frac{\partial}{\partial x_{0}} \equiv \frac{1}{\rho_{0}} \frac{\partial}{\partial x_{0}} .
$$

Thus, (4.59) can also be written in the form:

$$
\frac{\partial}{\partial t}\left[\rho_{0}\left(\frac{1}{2} u^{2}+\varepsilon \tau\right)\right]+\frac{\partial}{\partial x_{0}}(p u)=0 .
$$

Using

$$
I^{0}=\rho_{0}\left(\frac{1}{2} u^{2}+\varepsilon \tau\right), \quad I^{1}=p u,
$$

for the Lagrangian conserved density $I^{0}$ and flux $I^{1}$ in (4.60) and using the transformations for the conserved Eulerian density $F^{0}$ and flux components $F^{j}$ :

$$
F^{0}=\frac{I^{0}}{J}, \quad F^{j}=\frac{u^{j} I^{0}+x_{j k} I^{k}}{J}
$$

where $x_{j k}=\partial x^{j} / \partial x_{0}^{k}$ and $J=\operatorname{det}\left(x_{j k}\right)$ (Padhye and Morrison (1996a, b), Padhye (1998), Webb et al. (2005)) where $1 \leq j, k \leq n$ (note $n=1$ in our case), we obtain the Eulerian energy conservation law:

$$
\frac{\partial}{\partial t}\left[\frac{1}{2} \rho u^{2}+\varepsilon(\rho, S)\right]+\frac{\partial}{\partial x}\left[\rho u\left(\frac{1}{2} u^{2}+w\right)\right]=0
$$


where $w=(\varepsilon+p) / \rho$ is the gas enthalpy.

For the case $\beta=1$, the pullback conservation law (4.53) reduces to:

$$
\frac{\partial I^{0}}{\partial t}+\frac{\partial I^{1}}{\partial m}=\frac{\partial}{\partial t}\left(u \tau+r S_{m}\right)+\frac{\partial}{\partial m}\left(w-\frac{1}{2} u^{2}\right)=0 .
$$

This conservation law is due to translation invariance of the action with respect to $m$ in Noether's theorem. Translation in $m$ is a fluid relabelling symmetry. This is distinctly different than a translation in the Eulerian position variable $x$, which is associated with the conservation of linear momentum in Eulerian coordinates $(t, x)$ as independent variables. Using the transformations (4.62) for the case $n=1$ (i.e 1D gas dynamics), we obtain the Eulerian, version of the conservation law (4.64), namely:

$$
\frac{\partial}{\partial t}\left(u+r \frac{\partial S}{\partial x}\right)+\frac{\partial}{\partial x}\left(w+\frac{1}{2} u^{2}+u r \frac{\partial S}{\partial x}\right)=0 .
$$

Since $d r / d t=-T(x, t)$ then the Clebsch variable $r$ is a nonlocal variable, i.e.

$$
r(x, t)=-\int_{0}^{t} T\left(x, t^{\prime}\right) d t^{\prime}+r_{0}\left(\mathbf{x}_{0}\right),
$$

is minus the Lagrangian time integral of the temperature back along the fluid path $\left(r_{0}\left(\mathbf{x}_{0}\right)\right.$ is an 'integration constant'). By noting $d S / d t=(\partial / \partial t+u \partial x) S=0$ and using $d r / d t=-T$ equation (4.65) gives the Eulerian momentum equation for the fluid as:

$$
\frac{\partial u}{\partial t}+u \frac{\partial u}{\partial x}=-\frac{1}{\rho} \frac{\partial p}{\partial x} .
$$

Using the mass continuity equation (2.1), (4.67) can also be written in the momentum conservation form:

$$
\frac{\partial(\rho u)}{\partial t}+\frac{\partial}{\partial x}\left(\rho u^{2}+p\right)=0 .
$$

What is perhaps of most interest here, is the existence of a nonlocal conservation law (4.65) involving the nonlocal Clebsch potential $r(x, t)$ (see e.g. Webb et al. (2014a,b) for further nonlocal conservation laws involving a generalized nonlocal cross helicity conservation equation in MHD and a generalized nonlocal helicity conservation equation involving $r(\mathbf{x}, t)$ and the entropy $S$ of the fluid). 
The $m$-translation conservation law (4.65) is connected to the Clebsch variable formulation of fluid mechanics (e.g. Zakharov and Kuznetsov (1997), Morrison (1998), Webb et al. (2014c) ). In the Clebsch approach the fluid velocity $u$ has the form:

$$
u=\frac{\partial \phi}{\partial x}-r \frac{\partial S}{\partial x} \quad \text { where } \quad r=\frac{\beta}{\rho}
$$

Here $(\beta, S)$ and $(\rho, \phi)$ are canonically conjugate variables and $\phi$ is the velocity potential corresponding to potential flow (e.g. Zakharov and Kuznetsov (1997)). The potential $\phi$ satisfies Bernoulli's equation:

$$
\frac{\partial \phi}{\partial t}+u \frac{\partial \phi}{\partial x}=\frac{1}{2} u^{2}-w
$$

(4.65) has the conservation equation form:

$$
D_{t}+F_{x}=0
$$

in which:

$$
D=u+r \frac{\partial S}{\partial x}=\frac{\partial \phi}{\partial x}, \quad F=w+\frac{1}{2} u^{2}+u r \frac{\partial S}{\partial x}=-\frac{\partial \phi}{\partial t} .
$$

Thus, the conservation law (4.65), written in terms of the velocity potential $\phi$ reduces to the equation $\phi_{x t}-\phi_{t x}=0$, i.e. (4.65) can be written as the integrability condition $\phi_{x t}-\phi_{t x}=0$ for $\phi(x, t)$. Similarly, the Lagrangian form of the conservation law (4.64) can be written in the form: $\phi_{\theta m}-\phi_{m \theta}=0$ where $\partial / \partial \theta=\partial / \partial t+u \partial / \partial x$ is the Lagrangian time derivative following the flow. Note that

$$
\phi_{m}=\tau\left(u+r S_{x}\right) \quad \text { and } \quad \phi_{\theta}=\frac{1}{2} u^{2}-w
$$

\section{Symplecticity conservation law}

The symplecticity conservation law for the multi-symplectic system (4.48) is the set of conservation laws:

$$
D_{\alpha}\left(F_{\beta \gamma}^{\alpha}\right)=0, \quad \beta<\gamma
$$

where

$$
F_{\beta \gamma}^{\alpha}=\mathbf{z}_{, \beta}^{T} \mathrm{~K}^{\alpha} \mathbf{z}_{, \gamma} \equiv \mathrm{K}_{i j}^{\alpha} z_{, \beta}^{i} z_{, \gamma}^{j} .
$$


These conservation laws can be derived from the pullback (i.e. treat the dependent variables as functions of the independent variables) of the symplecticity conservation condition $\kappa_{, \alpha}^{\alpha}=$ 0 , where

$$
\kappa^{\alpha}=d \omega^{\alpha}=d\left(L_{j}^{\alpha} d z^{j}\right)=\frac{1}{2} K_{i j}^{\alpha} d z^{i} \wedge d z^{j},
$$

(Hydon (2005), see also Appendix A). The symplecticity conservation laws (4.74) are related to the pullback conservation laws (4.53) by the equations:

$$
D_{\gamma} G_{\beta}-D_{\beta} G_{\gamma}=D_{\alpha}\left(F_{\gamma \beta}^{\alpha}\right), \quad \gamma<\beta,
$$

where

$$
G_{\beta}=D_{\alpha}\left(L_{j}^{\alpha} z_{, \beta}^{j}-L \delta_{\beta}^{\alpha}\right) .
$$

Note that $G_{\beta}=0$ are the pullback conservation laws (4.53).

From (4.74)-(4.78), there is only one symplecticity conservation law in the present case. The pullback symplecticity conservation law (4.74) reduces to the equation:

$$
D_{\alpha}\left(F_{01}^{\alpha}\right)=D_{t}\left(u_{t} x_{m}-x_{t} u_{m}+r_{t} S_{m}\right)+D_{m}\left(p_{t} x_{m}-x_{t} p_{m}\right)=0 .
$$

The symplecticity conservation law (4.79) can be written in the form:

$$
\frac{\partial D}{\partial t}+\frac{\partial F}{\partial m}=\frac{\partial}{\partial t}\left[\frac{\partial(u, x)}{\partial(t, m)}+\frac{\partial(r, S)}{\partial(t, m)}\right]+\frac{\partial}{\partial m}\left(\frac{\partial(p, x)}{\partial(t, m)}\right)=0,
$$

where $\partial(\phi, \psi) / \partial(\alpha, \beta)$ is the Jacobian of $\phi$ and $\psi$ with respect to $\alpha$ and $\beta$. One can show that (4.80) can be written in the form:

$$
\frac{\partial D}{\partial t}+\frac{\partial F}{\partial m}=-\frac{\partial}{\partial m}\left[\frac{\partial}{\partial t}\left(\frac{1}{2} u^{2}+e\right)+\frac{\partial}{\partial m}(p u)\right]=0,
$$

where $e=\varepsilon \tau$ is the internal energy density of the gas per unit mass and $\tau=1 / \rho$. Equation (4.81) is minus the partial derivative with respect to $m$ of the comoving energy equation (4.58). The conserved density $D$ and flux $F$ in (4.80) can also be reduced to:

$$
D=-H_{m}, \quad F=H_{t},
$$


where $H=(1 / 2) u^{2}+w$ is the multi-symplectic Hamiltonian. Thus, the symplecticity conservation law (4.80) is also equivalent to the equation $-H_{m t}+H_{t m}=0$.

\section{Noether's theorem}

A multi-symplectic form of Noether's theorem was described by Webb et al. (2014c). The basic idea is that if the action:

$$
A=\iint L d m d t
$$

is invariant under the infinitesimal Lie transformations:

$$
m^{\prime}=m+\epsilon V^{m}, \quad t^{\prime}=t+\epsilon V^{t}, \quad z^{\prime s}=z^{s}+\epsilon V^{z^{s}},
$$

and under the divergence transformation:

$$
L^{\prime}=L+\epsilon D_{\alpha} \Lambda^{\alpha}
$$

then Noether's theorem for the multi-symplectic system implies the conservation law:

$$
D_{t}\left(V^{t} L+\hat{V}^{z^{s}} L_{s}^{0}+\Lambda^{0}\right)+D_{m}\left(V^{m} L+\hat{V}^{z^{s}} L_{s}^{1}+\Lambda^{1}\right)=0
$$

where

$$
\hat{V}^{z^{s}}=V^{z^{s}}-\left(V^{t} D_{t}+V^{m} D_{m}\right) z^{s}
$$

is the canonical or characteristic symmetry generator for transformations of $z^{s}$ of the form: $z^{\prime}=z^{s}+\epsilon \hat{V}^{z^{s}}, t^{\prime}=t, m^{\prime}=m$ that are equivalent to the transformations (4.84)-(4.85).

Example Consider the infinitesimal Lie transformations (4.84)-(4.85) with:

$$
V^{t}=0, \quad V^{m}=1, \quad V^{x}=0, \quad \Lambda^{\alpha}=0, \quad(\alpha=0,1)
$$

Here $\mathbf{z}=(x, u, p, S, r)^{T}$ (see (4.40) $)$. The action is invariant under the transformations with generators (4.88), and with the $L_{j}^{\alpha}$ given in (4.43) where $\omega^{\alpha}=L_{j}^{\alpha} d z^{j}$ and $L_{1}^{0}=u, L_{4}^{0}=r$, $L_{1}^{1}=p$ are the non-zero $L_{j}^{\alpha}$. Using (4.87) and (4.88) we obtain: $\hat{V}^{x}=-x_{m}, \hat{V}^{S}=-S_{m}$. 
The Noether conservation law (4.86) gives:

$$
D_{t}\left(\hat{V}^{x} u+\hat{V}^{S} r\right)+D_{m}\left(L+\hat{V}^{x} p\right)=0
$$

which reduces to the nonlocal conservation law (4.64). In other words, (4.64) follows from the $m$-translation invariance of the action (4.83) and Noether's theorem.

Similarly, the Lie transformations (4.84)-(4.85) with:

$$
V^{t}=1, \quad V^{m}=0, \quad V^{x}=0, \quad \Lambda^{0}=\Lambda^{1}=0,
$$

leaves the action (4.83) invariant under time translations and give rise to the energy conservation law (4.58). The transformations (4.84)-(4.85) with:

$$
V^{t}=0, \quad V^{m}=0, \quad V^{x}=1, \quad \Lambda^{0}=\Lambda^{1}=0,
$$

leave the action (4.83) invariant, corresponding to the $x$-translation invariance symmetry and give rise, via Noether's theorem to the $x$-momentum conservation law:

$$
u_{t}+p_{m}=0
$$

which is the Lagrangian form of the $x$-momentum conservation equation.

\section{E. Differential forms formulation}

Proposition IV.1. The condition that the action:

$$
J=\int \psi^{*}(\Theta) \equiv \int \tilde{L} d V
$$

is stationary, namely $\delta J / \delta z^{i}=0$ where $\Theta$ is the 2-form defined by the equations:

$$
\begin{aligned}
\Theta & =\omega^{\alpha} \wedge d \tilde{m}_{\alpha}-H d V, \\
d V & \left.=d t \wedge d m, \quad d \tilde{m}_{\alpha}=\partial_{\alpha}\right\lrcorner d V, \quad d \tilde{m}_{0}=d m, \quad d \tilde{m}_{1}=-d t,
\end{aligned}
$$


$\left(m^{0}=t\right.$ and $\left.m^{1}=m\right)$ gives the multi-symplectic system (4.48)-(4.51). In (4.93) $\tilde{L}$ is the multi-symplectic Lagrangian (4.9). We obtain:

$$
\begin{aligned}
\Theta & =\omega^{0} \wedge d m-\omega^{1} \wedge d t-H d t \wedge d m \\
& \equiv \pi_{x}^{t} d x \wedge d \tilde{m}_{0}+\pi_{S}^{t} d S \wedge d \tilde{m}_{0}+\pi_{x}^{m} d x \wedge d \tilde{m}_{1}-H d t \wedge d m \\
& \equiv(u d x+r d S) \wedge d m-p d x \wedge d t-H d V
\end{aligned}
$$

Hence the pullback of $\Theta$ is given by:

$$
\psi^{*}(\Theta)=\left(u x_{t}+r S_{t}+p x_{m}-H\right) d V \equiv\left(L_{s}^{\alpha} \frac{\partial z^{s}}{\partial m^{\alpha}}-H\right) d V=\tilde{L} d V
$$

Proof. The proof that $\delta J / \delta z^{i}=0$ gives the multi-symplectic system (4.51) follows by noting $\psi^{*}(\Theta)=\tilde{L} d V$ and using the analysis of Hydon (2005) to obtain:

$$
\frac{\delta J}{\delta z^{i}}=\frac{\partial \tilde{L}}{\partial z^{i}}-\frac{\partial}{\partial m^{j}}\left(\frac{\partial \tilde{L}}{\partial\left(\partial_{j} z^{i}\right)}\right)=\mathrm{K}_{i j}^{\alpha} \frac{\partial z^{j}}{\partial m^{\alpha}}-\frac{\partial H}{\partial z^{i}}=0
$$

Proposition IV.2. Consider the variational functional

$$
K[\Omega]=\int_{M} \Omega
$$

where $M$ is a region with boundary $\partial M$ in the fiber bundle space in which the $z^{i}$ are regarded as independent of the base manifold coordinates $m^{\alpha}=(t, m)$. The form:

$$
\Omega=d \Theta=d \omega^{\alpha} \wedge d \tilde{m}_{\alpha}-d H \wedge d V \quad \text { where } \quad d V=d t \wedge d m
$$

is known as the Cartan-Poincaré form. Consider variations of the functional (4.99) described by the Lie derivative:

$$
\mathcal{L}_{\mathbf{V}}=\frac{d}{d \epsilon}=V^{i} \frac{\partial}{\partial z^{i}}
$$

in which the base manifold variables $m^{\alpha}$ are fixed. The vector field $\mathbf{V}$ is an arbitrary but 
smooth vector field. The variations of $K[\Omega]$ described by:

$$
\delta K[\Omega]=\int_{M} \mathcal{L}_{\mathbf{V}}(\Omega)
$$

can be reduced to the form:

$$
\delta K[\Omega]=\int_{\partial M} V^{p} \beta_{p}
$$

where the forms $\left\{\beta_{p}: 1 \leq p \leq N\right\}$ ( $N$ is the number of $z^{i}$ variables) are given by:

$$
\beta_{p}=\mathrm{K}_{p j}^{\alpha} d z^{j} \wedge d \tilde{m}_{\alpha}-\frac{\partial H}{\partial z^{p}} d V
$$

and $\partial M$ is the boundary of the region $M$ in the $\mathbf{z}$-space. The set of equations $\beta_{p}=0$ $(1 \leq p \leq N)$, can be used as a basis of Cartan forms for the multi-symplectic system (4.48)-(4.51). The pullback of the differential forms $\beta_{p}=0$ to the base manifold gives the equations:

$$
\tilde{\beta}_{p}=\left(\mathrm{K}_{p j}^{\alpha} \frac{\partial z^{j}}{\partial m^{\alpha}}-\frac{\partial H}{\partial z^{p}}\right) d V .
$$

The sectioned forms $\tilde{\beta}_{p}$ vanish on the solution manifold of the multi-symplectic partial differential equation system (4.48)-(4.51).

Proof. To prove (4.103) from (4.102) we use Cartan's magic formula:

$$
\left.\left.\left.\mathcal{L}_{\mathbf{V}} \Omega=\mathbf{V}\right\lrcorner d \Omega+d(\mathbf{V}\lrcorner \Omega\right) \equiv d(\mathbf{V}\lrcorner \Omega\right),
$$

where we have used the fact that $\Omega=d \Theta$ and $d \Omega=d d \Theta=0$. Using (4.106) in (4.102) and using Stokes' theorem, we obtain:

$$
\left.\left.\delta K[\Omega]=\int_{M} d(\mathbf{V}\lrcorner \Omega\right)=\int_{\partial M}(\mathbf{V}\lrcorner \Omega\right) .
$$

Using (4.100) for $\Omega$ and noting that

$$
d \omega^{\alpha}=\frac{1}{2} \mathrm{~K}_{i j}^{\alpha} d z^{i} \wedge d z^{j}, \quad d H=\frac{\partial H}{\partial z^{i}} d z^{i} \wedge d V
$$

we obtain:

$$
\Omega=\frac{1}{2} \mathrm{~K}_{i j}^{\alpha} d z^{i} \wedge d z^{j} \wedge d \tilde{m}_{\alpha}-\frac{\partial H}{\partial z^{i}} d z^{i} \wedge d V .
$$


Thus,

$$
\begin{aligned}
\mathbf{V}\lrcorner \Omega & \left.=V^{p} \frac{\partial}{\partial z^{p}}\right\lrcorner\left(\frac{1}{2} \mathrm{~K}_{i j}^{\alpha} d z^{i} \wedge d z^{j} \wedge d \tilde{m}_{\alpha}-\frac{\partial H}{\partial z^{i}} d z^{i} \wedge d V\right) \\
& =V^{p}\left(\mathrm{~K}_{p j}^{\alpha} d z^{j} \wedge d \tilde{m}_{\alpha}-\frac{\partial H}{\partial z^{p}} d V\right)=V^{p} \beta_{p} .
\end{aligned}
$$

In deriving (4.110) we used the anti-symmetry of the matrices $\mathrm{K}_{i j}^{\alpha}$ and the antisymmetry of the wedge product to obtain the final result. Substituting (4.110) in (4.107) now gives (4.103) and (4.104) for $\delta K[\Omega]$. Note that the variational principle

$$
\delta K[\Omega]=\int_{\partial M} V^{p} \beta_{p}=0 \quad \text { implies } \quad \beta_{p}=0,
$$

for $1 \leq p \leq N$, since the $V^{p}$ are arbitrary smooth functions. The closure of the differential form system of equations $\beta_{p}=0$ in (4.111) is a set of Cartan forms representing the original multi-symplectic partial differential system (4.52) (see e.g. Harrison and Estabrook (1971)).

From (4.104) we obtain the pullback forms:

$$
\tilde{\beta}_{p}=\mathrm{K}_{p j}^{\alpha} \frac{\partial z^{j}}{\partial m^{s}} d m^{s} \wedge d \tilde{m}_{\alpha}-\frac{\partial H}{\partial z^{p}} d V .
$$

However, if there are $n$ spatial $m^{\alpha}$ components, then

$$
\begin{aligned}
d m^{s} \wedge d \tilde{m}_{\alpha} & =d m^{s} \wedge(-1)^{\alpha} d m^{0} \wedge \ldots \wedge d m^{\alpha-1} \wedge d m^{\alpha+1} \wedge \ldots d m^{n} \\
& =d m^{s} \delta_{\alpha}^{s}(-1)^{2 \alpha} d V=\delta_{\alpha}^{s} d V
\end{aligned}
$$

For 1D gas dynamics, $n=1$. Using (4.113), the pullback forms (4.112) reduce to the forms (4.105). This completes the proof.

In the case of 1D Lagrangian, compressible gas dynamics, the forms (4.104) reduce to:

$$
\beta_{p}=\mathrm{K}_{p j}^{0} d z^{j} \wedge d m-\mathrm{K}_{i j}^{1} d z^{j} \wedge d t-\frac{\partial H}{\partial z^{i}} d t \wedge d m, \quad 1 \leq p \leq 5
$$


The closure of the system of forms $\left\{\beta_{i}: \quad 1 \leq i \leq 5\right\}$, comprise a Cartan differential forms representation of multi-symplectic differential equation system (4.52). Sectioning the forms (i.e. applying the pullback to the forms) results in the system (4.52), i.e. $\tilde{\beta}_{i}=0$ gives (4.52), where the tilde means sectioning the forms.

The two-forms $\left\{\beta_{1}, \beta_{2}, \beta_{3}, \beta_{4}, \beta_{5}\right\}$ in (4.114) for the 1D gas dynamic system are:

$$
\begin{aligned}
& \beta_{1}=-d u \wedge d m+d p \wedge d t, \quad \beta_{2}=(d x-u d t) \wedge d m \\
& \beta_{3}=d t \wedge(d x-\tau d m), \quad \beta_{4}=-(d r+T d t) \wedge d m, \quad \beta_{5}=d S \wedge d m .
\end{aligned}
$$

In (4.115) the basic variables are $\mathbf{z}=(x, u, p, S, r)^{T}$. The specific volume $\tau$ and the temperature of the gas $T$ are functions of the enthalpy $\tilde{w}(p, S) \equiv w(\rho, S)$, and are given by the formulae:

$$
\tilde{w}_{p}=\tau, \quad \text { and } \quad \tilde{w}_{S}=T
$$

We now show, that the 2-forms (4.115) form a closed ideal of forms that represent the gas dynamics system (4.52) (e.g. Harrison and Estabrook (1971)). This means that the exterior derivatives of the basis forms $\left\{\beta_{i}: 1 \leq i \leq 5\right\}$ can be written as a combination of the basis forms (4.115) in the form:

$$
d \beta_{i}=c_{i j} \wedge \beta_{j}
$$

where the $c_{i j}$ are 1-forms (some of the $c_{i j}$ are in fact zero). Straightforward evaluation of $d \beta_{1}, d \beta_{2}$ and $d \beta_{5}$ give the equations:

$$
d \beta_{1}=0, \quad d \beta_{2}=\beta_{1} \wedge d t, \quad d \beta_{5}=0 .
$$

Using (4.117) gives:

$$
\begin{aligned}
& d \tau=d \tilde{w}_{p}=\tilde{w}_{p p} d p+\tilde{w}_{p S} d S \\
& d T=d \tilde{w}_{S}=\tilde{w}_{S p} d p+\tilde{w}_{S S} d S
\end{aligned}
$$


and using these results in (4.115) gives:

$$
\begin{aligned}
d \beta_{3} & =-\left(\tilde{w}_{p p} d p+\tilde{w}_{p S} d S\right) \wedge d t \wedge d m \\
& =-\tilde{w}_{p p} \beta_{1} \wedge d m+\tilde{w}_{p S} \beta_{5} \wedge d t .
\end{aligned}
$$

A similar calculation gives:

$$
d \beta_{4}=\tilde{w}_{S S} \beta_{5} \wedge d t-\tilde{w}_{S p} \beta_{1} \wedge d m .
$$

Equations (4.118), (4.120) and (4.121) demonstrates that the exterior derivatives of the forms $I=\left\{\beta_{1}, \beta_{2}, \beta_{3}, \beta_{4}, \beta_{5}\right\}$ lie in the ideal $I$, i.e. the set $I$ is a closed ideal of forms that represent the $1 \mathrm{D}$ gas dynamic equations (4.52). We can in fact, replace the 2 -forms $\beta_{1}$ and $\beta_{5}$ in the ideal $I$ by 1 -forms $\alpha_{1}$ and $\alpha_{5}$ by noting:

$$
\begin{aligned}
& \beta_{1}=d \alpha_{1} \quad \text { where } \quad \alpha_{1}=-u d m+p d t, \\
& \beta_{5}=d \alpha_{5} \quad \text { where } \quad \alpha_{5}=S d m .
\end{aligned}
$$

At this point we could proceed to obtain the Lie symmetries and conservation laws of (4.52) using the ideal $I$ and the methods of Harrison and Estabrook (1971) and Wahlquist and Estabrook (1975). However, this lies beyond the scope of the present paper, and will be investigated in a separate paper. Here, we merely note that for the case of an ideal gas with constant adiabatic index $\gamma$ with equation of state:

$$
p=p_{0}\left(\frac{\tau}{\tau_{0}}\right)^{-\gamma} \exp \left(\frac{S}{C_{v}}\right)
$$

the enthalpy $\tilde{w}(p, S)$ is given by:

$$
\tilde{w}(p, S)=\frac{a_{0}^{2}}{\gamma-1}\left(\frac{p}{p_{0}}\right)^{(\gamma-1) / \gamma} \exp \left(\frac{S}{\gamma C_{v}}\right)
$$

where $a_{0}^{2}=\gamma p_{0} / \rho_{0}$ is the square of the sound speed of the gas in 0-state. 


\section{SUMMARY AND CONCLUDING REMARKS}

In this paper we cast the equations of ideal, Lagrangian 1D fluid dynamics in a multisymplectic form (equations (4.49)-(4.51)). We used $m$ (the Lagrangian mass coordinate) and time $t$ as the independent variables. The dependent variables used were $\mathbf{z}=(x, u, p, S, r)^{T}$ where $x$ is the Eulerian particle position, $u=x_{t}$ is the fluid velocity, $p$ is the gas pressure, $S$ is the entropy and $r$ is the Clebsch variable or Lagrange multiplier in the modified Lagrangian which ensures $\partial S(m, t) / \partial t=0$. The equations are related to a multi-momentum, de DonderWeyl Hamiltonian formulation (Section 4.1) in which $u=x_{t}=\partial L / \partial x_{t}, p=\partial L / \partial x_{m}$ and $r=\partial L / \partial S_{t}$ are the momentum variables and $L$ is the Lagrangian. The same equations are also cast in a multi-symplectic form (4.49) -(4.51), in which the Hamiltonian $H=(1 / 2) u^{2}+$ $w \equiv F_{E} /(\rho u)$ is the ratio of the Eulerian energy flux $\left(F_{E}\right)$ to the mass flux $(\rho u)$ and $w=(\varepsilon+p) / \rho$ is the gas enthalpy.

We obtained the pullback conservation laws of the multisymplectic, Lagrangian gas dynamics system and the symplecticity conservation law (see e.g. Hydon (2005)). The pullback conservation laws can also be obtained from Noether's theorem for the multisymplectic system, and correspond to the invariance of the action under time translation (giving rise to the energy conservation law), and a conservation law associated with translations in $m$. The conservation law due to translations in $m$ is a fluid relabelling symmetry conservation law. It is a nonlocal conservation law, involving the nonlocal Clebsch variable $r$ which is the Lagrange multiplier used to ensure that $S$ is advected with the flow, (i.e. $S_{t}=0$ ). The variable $r$ satisfies the evolution equation $r_{t}=-T$ where $T$ is the temperature of the gas. The $m$-translation conservation law (4.64) or its Eulerian version (4.65) takes its most simple form by using the Clebsch representation for the fluid velocity, namely $u=\nabla \phi-r \nabla S$. Conservation law (4.65) reduces to the equation $\phi_{x t}-\phi_{t x}=0$. Thus, both the Eulerian Clebsch representation (e.g. Cotter et al. (2007), Webb et al. (2014c)) and the Lagrangian versions of the multi-symplectic fluid equations (this paper) are useful. The symplecticity law (4.80) is equivalent to a compatibility condition on the pullback conservation laws (see (4.77)). The symplecticity law (4.80) is equivalent to minus $\partial / \partial m$ operating on the co-moving energy equation (4.58). It is also equivalent to the the equation $H_{m t}-H_{t m}=0$ where $H=(1 / 2) u^{2}+w$ is the multi-symplectic Hamiltonian using the Lagrangian variables. 
The Cartan-Poincaré $(n+2)$-form $\Omega(n=1$ in $1 \mathrm{D}$ gas dynamics) gives rise via the variational principle (4.99)-(4.102), to a set of two-forms $I=\left\{\beta_{1}, \beta_{2}, \beta_{3}, \beta_{4}, \beta_{5}\right\}$ representing the $1 \mathrm{D}$ gas dynamics equations. The set $I$ is a closed ideal of forms, which may be used to determine the Lie symmetries and conservation laws of the system using Cartan's geometric theory of partial differential equations (e.g. Harrison and Estabrook (1971)). The Lie point symmetries and potential symmetries of the gas dynamic equations has been investigated by Akhatov et al. (1991), Cheviakov (2008), Bluman et al. (2006, 2010), and Sjöberg and Mahomed (2004). The potential symmetries can lead to nonlocal conservation laws of the system. Webb and Zank (2009) investigated conservation laws associated with the scaling symmetries of the 1D gas dynamic equations and with the nonlocal symmetry of the potential cover system of equations obtained by Siöberg and Mahomed (2004).

Webb et al. (2014c) studied the 1D multi-symplectic gas dynamic equations, by using Clebsch variables. In this paper, the multi-symplectic, Lagrangian gas dynamic equations were obtained. In general, we expect that there is a map between the Lagrangian and Eulerian multi-symplectic gas dynamic equations. This subject lies beyond the scope of the present paper. It is of interest to extend the present 1D analysis to the fully 3D compressible fluid dynamics and magnetohydrodynamics (MHD) equations, using for example the work of Newcomb (1962) who obtained variational principles for the MHD using the Lagrangian map, without using Clebsch variables. A recent comprehensive overview of multi-symplectic systems by Roman-Rou (2009) contains other points of the theory that have not been addressed in the present development. For example, Kanatchikov (1993, 1997, 1998) and Forger and Romero (2005) discuss the covariant Poisson brackets for multi-symplectic and de Donder-Weyl Hamiltonian systems (see also Marsden et al. (1986) who write the field equations in a single Poisson bracket form $\{F, S\}=0$ where $S$ is the action integral with applications to electrodynamics, relativistic Maxwell-Vlasov equations in plasma physics, and in general relativity).

\section{ACKNOWLEDGEMENTS}

I would like to thank the referee for a thorough report, that pointed out inconsistencies in the propositions IV.I and IV.2 in the original manuscript. I acknowledge discussions with Darryl Holm on fluid relabelling symmetries, Lagrangian fluid mechanics and multi- 
symplectic formulations of fluid mechanics. I acknowledge discussions with Phil. Morrison on multi-symplectic MHD. I thank Prof. QuanMing Lu (CAS, Key Lab. for Geospace environment, USTC, in Hefei, China) for financial support to attend the GAMP Geometric Algorithms and Methods in Plasma Physics Conference, Hefei, Anhui Province, May 13-15, 2014, where I presented work on Eulerian, multi-symplectic MHD using Clebsch variables. I acknowledge discussions with J. F. McKenzie and G.P. Zank.

\section{APPENDIX A}

In this appendix we indicate the derivation of the pullback conservation laws (4.53) and the symplecticity conservation laws (4.74)-(4.75) (see Hydon (2005) for more detail).

For multi-symplectic systems with associated 1-forms $\omega^{\alpha}=L_{j}^{\alpha} d z^{j}$, the equations are:

$$
\mathbf{K}_{i j}^{\alpha} \frac{\partial z^{j}}{\partial x^{\alpha}}=\frac{\partial H(\mathbf{z})}{\partial z^{i}}, \quad \mathbf{K}_{i j}^{\alpha}=\frac{\partial L_{j}^{\alpha}}{\partial z^{i}}-\frac{\partial L_{i}^{\alpha}}{\partial z^{j}}
$$

The generalized Legendre transformation for this system is:

$$
\left(L_{j}^{\alpha} d z^{j}\right)_{, \alpha}=d L
$$

where

$$
L=L_{j}^{\alpha} z_{, \alpha}^{j}-H(\mathbf{z})
$$

The pullback of (A.2) in which $d z^{j}=\left(\partial z^{j} / \partial x^{\beta}\right) d x^{\beta}$ and $d L=\left(\partial L / \partial x^{\beta}\right) d x^{\beta}$ gives the pullback conservation law (4.53). Similarly, the symplecticity condition:

$$
\kappa_{, \alpha}^{\alpha}=0 \quad \text { where } \quad \kappa^{\alpha}=d \omega^{\alpha}=\frac{1}{2} K_{i j}^{\alpha} d z^{i} \wedge d z^{j}
$$

pulled back to the base manifold with independent variables $x^{\beta}$, gives:

$$
\kappa_{, \alpha}^{\alpha}=\frac{1}{2}\left(\mathrm{~K}_{i j}^{\alpha} \frac{\partial z^{i}}{\partial x^{\beta}} \frac{\partial z^{j}}{\partial x^{\gamma}} d x^{\beta} \wedge d x^{\gamma}\right)_{, \alpha}=0
$$

which implies the symplecticity conservation laws (4.74)-(4.75). 


\section{REFERENCES}

Akhatov, I., Gazizov, R., and Ibragimov, N. nonlocal symmetries, heuristic approach (English translation), J. Sov. Math., 55 (1) 1401 (1991).

Bluman, G., Cheviakov, A. and Ivanova, N. Framework for nonlocally related partial differential equations systems and nonlocal symmetries: extension, simplifications and examples, J. Math. Phys., 47, 113505 (2006).

Bluman, G. W., Cheviakov, A.F. and Anco, S. Applications of Symmetry Methods to Partial Differential Equations, New York, NY:Springer (2010).

Bridges, T.J. 1992, Spatial Hamiltonian structure, energy flux and the water wave problem, Proc. Roy. Soc. Lond., A, 439, 297-315.

Bridges, T. J., Hydon, P.E. and Reich, S. Vorticity and symplecticity in Lagrangian fluid dynamics, J. Phys. A, 38, 1403-1418 (2005).

Bridges, T.J., Hydon, P.E. and Lawson, J.K. multi-symplectic structures and the variational bi-complex, Math. Proc. Camb. Phil. Soc., 148, 159-178 (2010).

Broer, L.J. F. and Kobussen, J.A., Conversion from material to local coordinates as a canonical transformation, Appl. Sci. Res., 29, 419-429 (1974).

Cantrijn, A., Ibort, A., and de León M. On the geometry of multisymplectic manifolds, J. Austral. Math. Soc. (Ser. A), 66, 303-330 (1999).

Carinena, J.F., Crampin, M. and Ibort, L.A., On the multi-symplectic formalism for first order field theories, Differential geometry and its applications, 1, 345-374 (North Holland) (1991).

Chandre, C., Casimir invariants and the Jacobi identity in Dirac's theory of constraints of constrained Hamiltonian systems, J. Phys. A., Math. and Theoret., 46, Issue 37, 375201 (2013a).

Chandre, C., de Guillebon, L., Back, A, Tassi, E. and Morrison, P. J. On the use of projectors for Hamiltonian systems and their relationship with Dirac brackets, J. Phys. A., Math. and Theoret., 46, Issue 12, 125203 (2013).

Cheviakov, A.F., An extended procedure for finding exact solutions of partial differential equations arising from potential symmetries. Applications to gas dynamics, J. Math. Phys., 49, 08352-1-18 (2008).

Courant, R. and Friedrichs, K.O. Supersonic Flow and Shock Waves, Springer-Verlag, New 
York, (1976).

Cotter, C.J., Holm, D.D. and Hydon, P.E., Multi-symplectic formulation of fluid dynamics using the inverse map, Proc. Roy. Soc. London, A, 463, 2617-2687 (2007).

de Donder Th., Théorie Invariantive du Calcul des Variations, (Gauthier Villars, Paris) (1935).

Forger, M., Paufler, C. and Römer, H., A general construction of Poisson brackets on exact multisymplectic manifolds, Reports on Math. Phys., 51, 187-195 (2003).

Forger, M. and Romero, S.V., Covariant Poisson brackets in geometric field theory, Commun. Math. Phys., 256, 375-410, Doi 10.1007/s00220-005-1287-8 (2005)

Gotay, M. J., A multi-symplectic framework for classical field theory and the Calculus of Variations I: covariant Hamiltonian formalism, in M. Francaviglia ed. Mechanics, Analysis and Geometry: 200 years after Lagrange (North Holland, Amsterdam 1991) 203-235 (1991a).

Gotay, M. J., A multisymplectic framework for classical field theory and the Calculus of Variations II: space+time decomposition, Differential Geometry and its Applications, 1, (1991), 375-390 (North Holland) (1991b)

Goldstein, H., Classical Mechanics, Addison Wesley, 2nd Edition, Reading Mass (1980).

Gotay, M. J., Isenberg, J., Marsden, J.E., Montgomery, (with J. Sniatycki and P.B. Yasskin collaborators), Momentum Maps and Classical Fields, Part I: Covariant Field Theory, arxiv:physics/9801019v2[math-ph], August 2004 (2004a).

Gotay, M. J., Isenberg, J., Marsden, J. E., (with R. Montgomery, J. Sniatycki and P.B. Yasskin collaborators) Momentum Maps and Classical Fields, Part II: Canonical Analysis of Field Theories, arXiv:math-ph/0411032v1, 9 Nov. 2004b, (2004b).

Harrison, B. K. and Estabrook, F. B., Geometric approach to invariance groups and solution of partial differential systems, J. Math. Phys., 12, 653-66 (1971).

Holm, B. A. and Kupershmidt, 1983a, Poisson brackets and Clebsch representations for magnetohydrodynamics, multi-fluid plasmas and eleasticity, Physica D, 6D, 347-63.

Holm, B. A. and Kupershmidt, 1983b, Noncanonical Hamiltonian formulation of magnetohydrodynamics, Physica D, D7, 330-3.

Holm, D. D., Marsden, J.E. and Ratiu, T.S., The Euler-Lagrange equations and semiproducts with application to continuum theories, Advances in Math., 137, (1), 1-81 (1998).

Hydon, P. E., Multisymplectic conservation laws for differential and differential-difference 
equations, Proc. Roy. Soc. A, 461, 1627-1637 (2005).

Kanatchikov, I. V., On the canonical structure of the de-Donder-Weyl covariant Hamiltonian formulation of field theory I. Graded Poisson brackets and equations of motion, preprint arxiv:hep-th/9312162v1, 20th Dec. 1993 (1993).

Kanatchikov, I. V., On field theoretic generalizations of a Poisson algebra, Rep. Math. Phys., 40, (1997), 225-234, hep-th/9710067 (1997).

Kanatchikov, I. V., Canonical structure of classical field theory in the polymomentum phase space, Rep. Math. Phys., 41, (1998), 49-90, hep-th/9709229 (1998).

Marsden, J. E., Montgomery, R., Morrison, P.J., and Thompson, W.B., Covariant Poisson brackets for classical fields, Annals of Physics, 169, 29-47 (1986).

Marsden, J.E. and Shkoller, S., Multi-symplectic geometry, covariant Hamiltonians and water waves, Math. Proc. Camb. Phil. Soc., 125, 553-575 (1999).

Morrison, P.J., Hamiltonian description of the ideal fluid, Rev. Mod. Phys., 70, (2), 467-521 (1998).

Newcomb, W.A., Lagrangian and Hamiltonian methods in magnetohydrodynamics, Nucl. Fusion Suppl., Part 2, 451-463(1962).

Nutku, Y. 1987, On a new class of completely integrable systems II. Multi-Hamiltonian structure, J. Math. Phys., 28, 2579.

Olver, P. J. and Nutku, Y. 1988, Hamiltonian structures for systems of hyperbolic conservation laws, J. Math. Phys., 29, 1610.

Padhye, N. and Morrison, P.J., Fluid element relabeling symmetry, Phys. Lett., A, 219, 287-292 (1996a).

Padhye, N. and Morrison, P.J., Relabeling symmetries in hydrodynamics and magnetohydrodynamics, Plasma Physics Reports, 22,(10), 869-877 (1996b).

Padhye, N.S., Topics in Lagrangian and Hamiltonian fluid dynamics: relabeling symmetry and ion acoustic wave stability, Ph. D. Dissertation, University of Texas at Austin (1998).

Paufler, C. and Römer, H. 2002, De Donder-Weyl equations and multi-symplectic geometry, Reports on Mathematical Physics, 49, 325-334, (Invited lecture of the XXXIII Symposium on Mathematical Physics, June 5-9, 2001, delivered by H. Römer.

Roman-Roy, N., Multi-symplectic Lagrangian and Hamiltonian formalisms of classical field theories, SIGMA, 5, 100 (25pp) (2009).

Sjöberg, A. and Mahomed, F.M., Non-local symmetries and conservation laws for one- 
dimensional gas dynamics equations, Appl. Math. and Computation, 150, 379-397 (2004).

Verosky, J. M. 1984, Higher order symmetries of the compressible one dimensional isentropic fluid equations, J. Math. Phys., 25 (4), april 1984, p. 884-

Wahlquist, H. D. and Estabrook, F.B., Prolongation structures of nonlinear evolution equations, J. Math. Phys., 16, p. 1-7 (1975).

Webb, G.M., Zank, G.P., Kaghashvili, E. Kh. and Ratkiewicz, R.E., Magnetohydrodynamic waves in non-uniform flows II: stress energy tensors, conservation laws and Lie symmetries, J. Plasma Phys., 71, 811-857, doi:10.1017/s))223778050003740 (2005).

Webb, G.M., McKenzie, J.F., Dubinin, E.M. and Sauer, K. 2005, Hamiltonian formulation of nonlinear travelling whistler waves, Nonl. Proc. Geophys., 12, 634-660.

Webb, G. M., McKenzie, J.F., Mace, R. L., Ko, C.M. and Zank, G.P. 2007, Dual variational principles for nonlinear traveling waves in multi-fluid plasmas, Phy. Plasmas, 4(8), 082318082318-17, doi:10.1063/1.2757154.

Webb, G.M., Ko, C.M., Mace, R.L., McKenzie, J.F. and Zank, G.P. 2008, Integrable, oblique travelling waves in charge neutral, two-fluid plasmas, Nonl. Proc. Geophys., 15, 179-208.

Webb, G. M. and Zank, G.P., Scaling symmetries, conservation laws and action principles in one-dimensional gas dynamics, J. Phys. A, Math. and Theor., 42, 475205 (23pp), doi:10.1088/1751-8113/42/47/475205 (2009).

Webb, G. M., Dasgupta, B., McKenzie, J.F., Hu, Q., and Zank, G.P., Local and nonlocal advected invariants and helicities in magnetohydrodynamics and gas dynamics, I, Lie dragging approach, J. Phys. A Math. and Theoret., 47, 095501 (33pp), doi:10.1088/17518113/49/095501, preprint at http://arxiv.org/abs/1307.1105 (2014a).

Webb, G. M., Dasgupta, B., McKenzie, J.F., Hu, Q., and Zank, G.P., Local and nonlocal advected invariants and helicities in magnetohydrodynamics and gas dynamics, II, Noether's theorems and Casimirs, J. Phys. A Math. and Theoret., 47, 095502 (31pp), doi:10.1088/1751-8113/49/095502, preprint at http://arxiv.org/abs/1307.1038 (2014b).

Webb, G.M., McKenzie, J.F. and Zank, G.P. Multi-symplectic magnetohydrodynamics, J. Plasma Phys., 80, pt. 5, pp. 707-743, (37pp), doi:10.1017/S0022377814000257 (2014c), preprint: http://ariv.org/abs1312.4890, (2014c)

Webb, G.M., Burrows, R.H., Ao, X. and Zank, G.P. Ion acoustic traveling waves, J. Plasma Phys., 80, part 2, pp. 147-171, doi:10.1017/S0022377813001013, preprint at http://ariv.org/abs/1312.6406 (2014d). 
Webb, G. M. and Mace, R.L., Potential vorticity in magnetohydrodynamics, J. Plasma Phys., 90581011S, doi:10.1017/S0022377814000658., preprint: http://arxiv/org/abs/1403.3133 (2014).

Weyl, H., Geodesic fields in the Calculus of Variation for multiple integrals, Annals of Math., 36, No. 3 (July 1935), pp. 607-629 (1935). http:/www.jstor.org/stable/1968645

Zakharov, V.E. and Kuznetsov, E.A., Reviews of topical problems: Hamiltonian formalism for nonlinear waves, Uspekhi, 40, 1087-116 (1997). 